\title{
STBC-assisted OFDM with Subcarrier Power Modulation
}

\author{
ABDELRAHMAN M.A. ABUQAMAR ${ }^{1}$, JEHAD HAMAMREH ${ }^{2}$, MOHAMEDOU ABEWA ${ }^{3}$ \\ ${ }_{1,2,3}$ The authors are with WISLAB for Wireless Research at the department of Electrical-Electronics Engineering, Antalya Bilim University, Antalya, Turkey. \\ Corresponding authors: A. ABUQAMAR and J. Hamamreh (e-mail: jehad.hamamreh@antalya.edu.tr). \\ The Matlab simulation codes used to generate the results in this paper can be found at https://researcherstore.com
}

\begin{abstract}
Orthogonal Frequency Division Multiplexing with Subcarrier-Power Modulation (OFDM-SPM) has recently been proposed as a promising, potential transmission technique for future wireless communications (i.e., 6G and beyond) due to its multiple beneficial characteristics, including higher spectral efficiency, low latency with good reliability while maintaining low complexity. In this paper, the OFDM-SPM technique is compounded with Alamouti space-time block coding (STBC) in a multiple-input-single-output (MISO) set up to study, investigate and quantify the wireless system's performance of their combination over a multipath Rayleigh fading channel. Particularly, we analyze the two main performance metrics, bit-error-rate (BER) and throughput of the newly designed technique called OFDM-SPM-STBC, and then quantify the amount of gains in both BER and throughput. For making STBC work well with OFDM-SPM, a suitable equalizer is proposed for detecting the SPM power bits, and it is observed that there is a considerable improvement in the BER performance. We also consider two scenarios for OFDM-SPM-STBC transmission. The first one is the power reassignment policy (PRP), and the second one is the power saving policy (PSP). It is found that the sub-carrier optimized power-reassignment scheme provides the best BER performance for the proposed transmission scheme. In contrast, the PSP for SPM provided an intermediate improvement in an average BER.
\end{abstract}

INDEX TERMS Alamouti STBC, BER, Equalizer, OFDM, Throughput, Subcarrier Power Modulation.

\section{INTRODUCTION}

$\mathbf{T}$ HE demands a higher data rate is increasing day by day. The radio spectrum is becoming more crowded; thus, there is a considerable need to design and develop techniques capable of providing higher spectrum efficiency. Orthogonal frequency division multiplexing (OFDM) is a widely accepted technique for transmission in many communication systems such as Wi-Fi, Wi-MAX, LiFi, LTE, and even 5G [1]. OFDM supports high data rate traffic by dividing the incoming serial data stream into parallel narrow-band channels. The fading channel becomes frequency independent and flat while minimizing the co-channel and adjacent channel interference. However, with the new requirements generated by the $5 \mathrm{G}+$ and $6 \mathrm{G}$ communication technologies and the spread of data-hungry applications, there is a need to add new degrees of freedom to the existing OFDM technique to improve its performance.
There have been many techniques proposed in the literature to improve the spectral efficiency of OFDM; among these methods, a new modulation technique named OFDM with sub-carrier power modulation (OFDM-SPM) has recently been proposed in [2]. The authors proposed utilizing the power of each sub-carrier to transmit additional information bits besides those transmitted by conventional modulation schemes such as M-ary PSK. It is shown that OFDM with SPM provides higher special efficiency along with the reduction in transmission power. It is also established that this technique maintains the gains when used with higherorder constellations [19].

In [4], OFDM-SPM is exhibited as a modulation technique capable of utilizing a third dimension (power) to convey extra data bits. OFDM-SPM manipulates the power of the subcarriers of an OFDM block and uses it as an additional degree of freedom to convey extra data bits. Also, the author in [5] proposes a non-coherent modulation scheme called orthogonal frequency division multiplexing with sub- 
carrier power modulation and differential phase-shift keying (OFDM-SPM-DPSK), as an effective modulation technique for future $6 \mathrm{G}$ and beyond systems. The proposed technique has the potential to reduce complexity, enhance power-saving while improving spectral efficiency significantly.

In addition, in [6] the concept of OFDM-SPM is extended to the case of using two-dimensional signal constellations that consist of both in-phase and quadrature components such as QPSK Symbol modulation. Moreover, in [7] the results show that compared to other proposed modulation techniques, which add a third dimension to carry data, OFDMSPM vastly improves spectral efficiency, where it is capable of doubling the spectral efficiency in addition to reducing the transmission power of the system by half. In [23], the author demonstrates and proves in this work the ability of the proposed two-dimensional modulation scheme termed as non-coherent orthogonal frequency division multiplexing with subcarrier power modulation and differential phase-shift keying in achieving the performance of a coherent design while reducing complexity. In [24] the results of OFDMSPM with QPSK were compared to that of conventional OFDM with 16-QAM symbol modulation. OFDM-SPM displayed superiority both in terms of BER and throughput, achieving a gain of approximately 2.5-3 dB. In [25] the author analyzes the drawbacks in conventional NOMA and presents a new kind of NOMA with more improved performance metrics. Furthermore, in [26] the proposed model uses the wireless channel characteristics to eliminate user interference as well as completely degrade the received signal at the eavesdropper's terminal. Moreover, In [27] the new proposed NOMA systems are motivated by the many limitations faced by current NOMA-based systems.

However, in OFDM-SPM, the power of each sub-carrier is used to transmit extra bits along with the bits transmitted by conventional symbol modulation schemes in OFDM. In this way, it helps improve spectral efficiency along with an additional gain of reducing and saving the transmission power. Although the original OFDM-SPM technique provides a marginal improvement in the overall BER in the power reassignment mode, the bit error rate of the additional bit-stream conveyed through the power levels is still higher than the other data stream sent through the conventional modulation constellation.

On the other hand, it is well-known that the transmit diversity scheme proposed by Alamouti in [3], Which utilizes space-time-block coding (STBC), can improve the BER of the signal in multipath fading channels. Space-time coding realizes spatial diversity by introducing temporal and spatial correlation into the signals transmitted from different transmits antennas. It has received increased attention due to improved hardware technologies and their potential to provide increased capacity for next-generation wireless systems. STBC-OFDM system under Rayleigh fading channel with known and unknown CSI at the transmitter is analyzed in [8], where the authors use the singular value decomposition (SVD) method for improving the BER performance and providing the CSI knowledge at the transmitter.; however, the SVD of the channel adds considerable computational complexity to the system.

STBC-OFDM system under Rayleigh fading channel with known and unknown CSI at the transmitter is analyzed in [8], where the authors use the singular value decomposition (SVD) method for improving the BER performance and providing the CSI knowledge at the transmitter.; however, the SVD of the channel adds considerable computational complexity to the system.

\section{A. NOVELTY AND BENEFITS OF THE PROPOSED ALGORITHM}

Cellular networks, wireless mesh networks (WMNs), wireless local area networks (WLANs), personal area networks (PANs), and wireless sensor networks (WSNs) are generally utilized nowadays. The growing demand for these networks has made the spectrum a valuable resource. There will always be a need for approaches to carry other bits per $\mathrm{Hz}$ for such a purpose. A specific solution that has now attracted much attention is to use multiple input single output MISO systems for increasing the capacity dates back to winters in [12] [13]. Space-Time block coding (STBC) has many advantages, as shown below:

- Beamforming: A pair of transmitter-receiver can beamform and direct their main beams to each other, thus increasing the received power of the receiver and thus the SNR.

- Spatial diversity: Through the transmission antennas, a signal can be coded, generating redundancy, where it reduces the probability of outage.

- Spatial multiplexing: A collection of streams is transmitted in parallel, or each stream is transmitted from a different antenna to split the signals. In this way, the receiver will perform the perfect signal processing.

To produce spatial diversity, space-time block coding can be used, where it allows a system to have an improved reliability performance in a wireless fading environment. Great performance with low complexity for decoding is feasible, where a maximum gain can be achieved.

However, the implementation of simple OFDM-SPM and STBC for higher constellation provides much promise to enhance the spectral efficiency performance of the system, which has not been investigated in the literature yet. Hence, analyzing the performance of OFDM-SPM along with STBC for improving spectral efficiency is the subject of this paper. The rest of the article is organized as follows.

The remainder of this paper is organized as follows. Section II presents the system model and problem formulation of OFDM-SPM-STBC. The methodology and performance analysis are carried out in Section III. Section IV presents a discussion on BER, whereas Section V presents the results and discussion. Section VI discusses the conclusion and possible future enhancements of the presented work in this paper. 


\section{SYSTEM MODEL}

This section discusses the details of the transmitter, multipath channel, and receiver design, which are considered in the analysis of the proposed OFDM-SPM-STBC scheme.

\section{A. TRANSMITTER DESIGN}

The implementation of OFDM with sub-carrier power modulation is presented in detail in [2]. The block diagram of the implemented transmitter design is shown in Fig. 1

In the transmitter, the incoming data bits are generated and are divided into two halves to implement the traditional modulation and sub-carrier power modulation. The optimal values of the high and low power levels, which reduce the scheme's BER, are found through exhaustive trial and error experiments [4]. The high and low power levels $H$ and $L$ are subject to Eq. (1) in power saving policy and to Eq. (2) in the power reassignment policy [21].

$$
\begin{aligned}
& L^{2}+H^{2}=2 E_{b} \\
& L^{2}+H^{2}=4 E_{b}
\end{aligned}
$$

By setting the value of $H$ to an arbitrary value, the corresponding value of $L$ can be found as follows, for PSP and PRP respectively:

$$
\begin{aligned}
& L=\sqrt{2 E_{b}-H^{2}} \\
& L=\sqrt{4 E_{b}-H^{2}}
\end{aligned}
$$

The optimal high and low levels for each power policy are indicated in TABLE 1.

TABLE 1: Optimal Power levels $(H, L)$.

\begin{tabular}{l|c|r} 
& $H$ & $L$ \\
\hline Power Saving Policy & 1.35 & 0.4213 \\
\hline Power Reassignment Policy & 1.918 & 0.5668 \\
\hline
\end{tabular}

After the modulation, the data is given to the STBC encoder. Alamouti STBC is used because it is the only STBC code that achieves the maximum diversity gain [14]. The Alamouti-STBC encoder generates two symbols streams such that:

$$
\left[\begin{array}{ll}
d_{1, t}(k) & d_{1, t+1}(k) \\
d_{2, t}(k) & d_{2, t+1}(k)
\end{array}\right]=\left[\begin{array}{cc}
x_{t}(k) & x_{t+1}^{*}(k) \\
x_{t+1}(k) & -x_{t}^{*}(k)
\end{array}\right],
$$

where $d(k)=a_{i}(k)+i b_{j}(k)$ denotes the sub-carrier modulation symbol, $x^{*}$ denotes the complex conjugate of $x$ and $k=(0,1, \ldots \ldots .,(N-1))$, where $N$ is the number of subcarriers. These streams are given as an input to two inverse fast Fourier transform (IFFT) blocks corresponding to two transmit antennas. The null sub-carriers are added before performing IFFT to the above-generated symbols such that the length of data becomes equal to the length of IFFT. Another purpose of adding the null-subcarriers is to synchronize the OFDM symbols at the receiver [15]. Moreover, in null subcarriers, no data symbols are transmitted and hence, no power, but they still have sub-carriers for channel estimation.
Before adding the cyclic prefix, the stream for antenna 1 and antenna 2 becomes:

$$
X_{l, m}(n)=\frac{1}{N} \sum_{k=0}^{N-1} x_{l, m}(k) W_{N}^{-n k},
$$

where $W_{N}^{m}=e^{j 2 \pi \frac{m}{N}}$, we take value of $l=1$ for antenna-1, representing row-1 of the matrix of Eq. (5) and $l=2$ for antenna-2, which is represented by row- 2 of the matrix of Eq.(5). To mitigate the effect of multipath, a cyclic prefix is added; this helps in mitigating the effect of intersymbol-interference (ISI) because OFDM has a long symbol duration; therefore, ISI only affects the initial part of the OFDM symbol [16] [17]. Hence, two STBC-OFDM-SPM streams are transmitted by two antennas from the transmitter, as shown in Fig 1 .

\section{B. CHANNEL MODEL}

We consider the Rayleigh multi-path fading channel with Ttaps, which are considered exponentially decaying taps. Let our multi-path Rayleigh faded channel be represented by $h$ such that [18]:

$$
h=\left[h_{0}, h_{1}, h_{2}, \ldots \ldots, h_{(\tau-1)}\right] .
$$

For two-transmit antennas and one receive antenna, two Rayleigh multi-path fading channels are considered. The channel from antenna- 1 and antenna- 2 to the receiver antenna, respectively, are defined as in the first and second row of Eq. (8) below:

$$
\left\{\begin{array}{l}
h_{1}=\left[h_{1,0}, h_{1,1}, h_{1,2}, \ldots \ldots, h_{1(\tau-1)}\right], \text { Antenna }-1 \\
h_{2}=\left[h_{2,0}, h_{2,1}, h_{2,2}, \ldots \ldots, h_{2(\tau-1)}\right], \text { Antenna }-2
\end{array}\right.
$$

Converting to the frequency domain, the channel response becomes:

$$
\left\{\begin{array}{l}
h_{F, 1}=F F T\left(h_{1}\right), \\
h_{F, 2}=F F T\left(h_{2}\right) .
\end{array}\right.
$$

We also compute the frequency domain conjugate channels as:

$$
\left\{\begin{array}{l}
h_{F, 1}^{1}=F F T\left(h_{1}^{*}\right), \\
h_{F, 2}^{2}=F F T\left(h_{2}^{*}\right) .
\end{array}\right.
$$

These conjugate frequency-domain responses would be later used in the receiver section for equalization purposes. If the received signal at the receiver is denoted by $y$, then it can be expressed as:

$$
y=h_{1} \otimes X_{1}+h_{2} \otimes X_{2}+\eta_{0},
$$

where $\otimes$ represents the convolution operation and $X_{i}$ represents the stream of OFDM symbols transmitted by the $i^{t h}$ antenna. $\eta_{0}$ is the AWGN noise. The AWGN noise is added and controlled by varying the SNR values from $0 \mathrm{~dB}$ to 35 $\mathrm{dB}[4]$. 


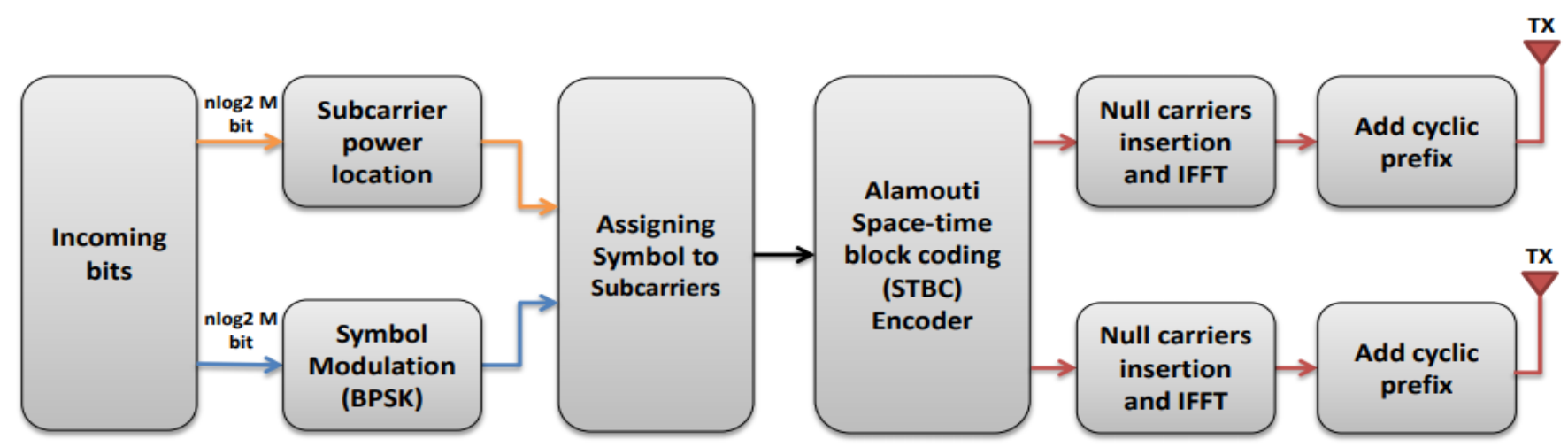

FIGURE 1: Transmitter design of OFDM-SPM with Alamouti STBC.

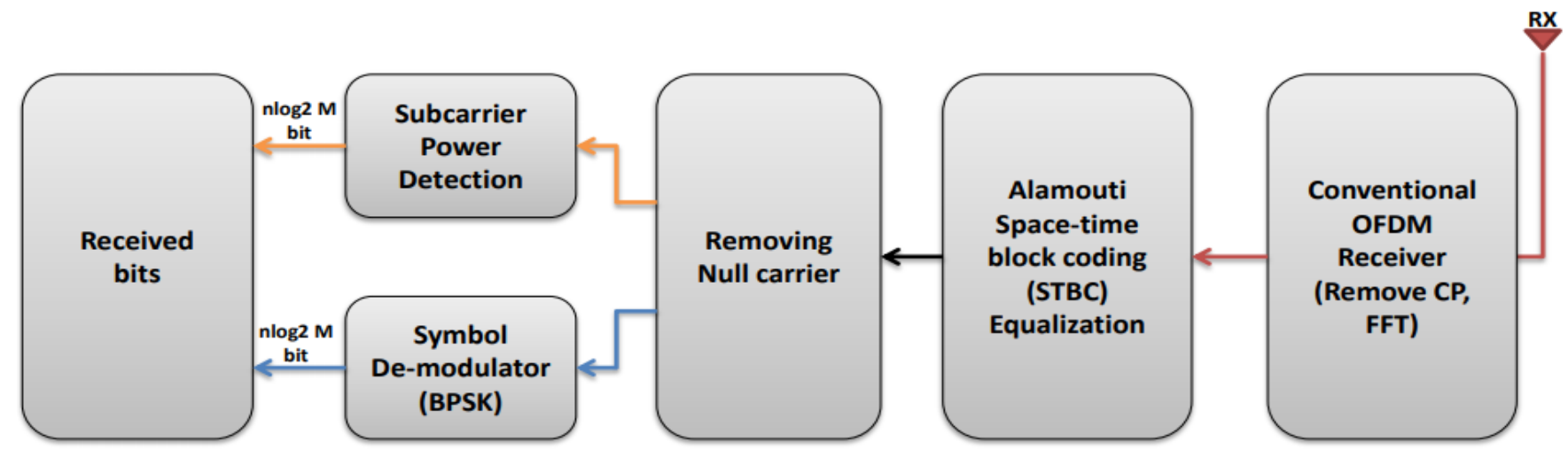

FIGURE 2: Receiver design of OFDM-SPM with Alamouti STBC.

\section{RECEIVER DESIGN}

The receiver design implementation for OFDM-SPM-STBC is shown in Fig.2. OFDM-SPM-STBC is capable of improving the BER and enhancing the spectral efficiency of the communication system. At the receiver, the initial steps of the traditional OFDM receivers are followed. The CP is removed from the received signal, and frequency domain STBC equalization is performed. The null sub-carriers are removed, and the demodulation of the signal bits and power bits are performed in parallel as described in [2]. The central part of the receiver section is the design of the OFDMSPM-STBC equalizer. The details of the implementation of the OFDM-SPM-STBC equalizer are provided in the next section.

\section{PROPOSED OFDM-SPM-STBC EQUALIZER}

To restore the signal at the receiver and combat the channel effects, we need to use an equalizer. In normal AlamoutiSTBC, we use the combining scheme [3] and the maximum likelihood decision rule, which reproduces the signal at the receiver. The received signal at the receiver in the form of matrix notation is described as:

$$
\left[\begin{array}{ll}
y_{t} & y_{t+1}
\end{array}\right]=\left[\begin{array}{ll}
h_{1} & h_{2}
\end{array}\right]\left[\begin{array}{cc}
x_{1} & -x_{2}^{*} \\
x_{2} & x_{1}^{*}
\end{array}\right]+\left[\begin{array}{ll}
n_{t} & n_{t+1}
\end{array}\right] .
$$

After the removal of the cyclic prefix, the traditional FFT operation on OFDM symbols is performed. Hence, after FFT operation, the input to the ODFM-SPM-STBC equalizer is given as:

$$
\left\{\begin{array}{l}
F\left[y_{t}(k)\right]=F\left[h_{1}\right] F\left[x_{1}\right]+F\left[h_{2}\right] F\left[x_{2}\right]+F\left[n_{t}\right], \\
F\left[y_{t+1}(k)\right]=F\left[h_{1}\right] F\left[\left(-x_{2}^{*}\right)\right]+F\left[h_{2}\right] F\left[\left(x_{1}^{*}\right)\right]+F\left[n_{t+1}\right] .
\end{array}\right.
$$

Where here, F [-] represents the Fourier transform of the respective function or signal. Using the frequency domain response notations of the channels as described in the channel model, we can re-write the above equations as:

$$
\left\{\begin{array}{l}
F\left[y_{t}(k)\right]=h_{F, 1} F\left[x_{1}\right]+h_{F, 2} F\left[x_{2}\right]+F\left[n_{t}\right], \\
F\left[y_{t+1}(k)\right]=h_{F, 1} F\left[\left(-x_{2}^{*}\right)\right]+h_{F, 2} F\left[\left(x_{1}^{*}\right)\right]+F\left[n_{t+1}\right] .
\end{array}\right.
$$

By dividing the first equation in the system defined in 14 by $h_{F, 1}$ and taking the conjugate of the second equation in 14 and dividing it by $h_{F, 2}^{2}$, we get:

$$
\left\{\begin{array}{l}
\frac{F\left[y_{t}(k)\right]}{h_{F, 1}}=F\left[x_{1}\right]+\frac{h_{F, 2} F[x 2]}{h_{F, 1}}+\frac{F\left[n_{t}\right]}{h_{F, 1}}, \\
\frac{F^{*}\left[y_{t+1}(k)\right]}{h_{F, 2}^{2}}=\frac{h_{F, 1}^{1} F^{*}\left[\left(-x 2^{*}\right]\right.}{h_{F, 2}^{2}}+F^{*}\left[\left(-x_{1}^{*}\right)\right]+\frac{F^{*}\left[n_{t+1}\right]}{h_{F, 2}^{2}} .
\end{array}\right.
$$


Using the conjugate-symmetry property of the Fourier transform for the second equation in the system of equations defined above, we can rewrite the system of equations as follows:

$$
\left\{\begin{array}{l}
\frac{F\left[y_{t}(k)\right]}{h_{F, 1}}=F\left[x_{1}\right]+\frac{h_{F, 2} F[x 2]}{h_{F, 1}}+\frac{F\left[n_{t}\right]}{h_{F, 1}}, \\
\frac{F\left[y_{t+1}^{*}(k)\right]}{h_{F, 2}^{2}}=\frac{h_{F, 1}^{1} F\left[\left(-x_{2}\right]\right.}{h_{F, 2}^{2}}+F\left[\left(x_{1}\right)\right]+\frac{F\left[n_{t+1}^{*}\right]}{h_{F, 2}^{*}} .
\end{array}\right.
$$

Subtracting the second equation form the first in the system (16), we get:

$\frac{F\left[y_{t}(k)\right]}{h_{F, 1}}-\frac{F\left[y_{t+1}^{*}(k)\right]}{h_{F, 2}^{2}}=\frac{h_{F, 2} F\left[x_{2}\right]}{h_{F, 1}}+\frac{h_{F, 2}^{1} F\left[x_{2}\right]}{h_{F, 2}^{2}}+N_{1}(f)$,

where $N_{1}(f)=\frac{F\left[n_{t}\right]}{h_{F, 1}}-\frac{F\left[n_{t+1}^{*}\right]}{h_{F, 2}^{2}}$. In this way, we find the OFDM symbol $X_{2}(k)=F\left[x_{2}(k)\right]$ from the equation (17).

Similarly, if the equation in the first row of (15) is divided by $h_{F, 2}$ and the conjugate of the second row is taken and divided by $h_{F, 1}^{1}$, we obtain the following result due to the conjugate-symmetry property of Fourier transform:

$$
\left\{\begin{array}{l}
\frac{F[y t(k)]}{h_{F, 2}}=\frac{h_{F, 1} F\left[x_{1}\right]}{h_{F, 2}}+F\left[x_{2}\right]+\frac{F\left[n_{t}\right]}{h_{F, 2}}, \\
\frac{F\left[y_{t+1}^{*}(k)\right]}{h_{F, 1}^{1}}=F\left[\left(-x_{2}\right)\right]+\frac{h_{F, 2}^{2} F^{*}[(-x 1)]}{h_{F, 1}^{1}}+\frac{F\left[n_{t+1}^{*}\right]}{h_{F, 1}^{1}} .
\end{array}\right.
$$

Adding the above two equations we get,

$\frac{F\left[y_{t(k)}\right]}{h_{F, 2}}+\frac{F\left[y_{t+1}^{*}(k)\right]}{h_{F, 1}^{1}}=\frac{h_{F, 1} F\left[x_{1}\right]}{h_{F, 2}}+\frac{h_{F, 2}^{2} F\left[x_{1}\right]}{h_{F, 1}^{1}}+N_{2}(f)$,

where $N_{2}(f)=\frac{F\left[n_{t}\right]}{h_{F, 2}}+\frac{F\left[n_{t+1}^{*}\right]}{h_{F, 1}^{1}}$. From Eq. 19, we get the OFDM symbol $X_{1}(k)=F\left[x_{1}(k)\right]$.

The equalizer for OFDM-SPM-STBC is defined by Eq. (17) and (19). At the output of this proposed OFDM-SPMSTBC equalizer, the null carriers are removed, and the raw bits are detected. After removing null carriers, the data stream is provided as an input to the two detectors, one for BPSK modulated data bits detection and the other for sub-carrier power modulated bits detection. After this detection process, the obtained bits are compared with the transmitted bits initially, and then the bit-error rate (BER) is computed.

\begin{tabular}{|c|c|}
\hline symbol & Description \\
\hline$y_{t} y_{t+1}$ & The received symbol on the first and second time slot \\
\hline$h_{1}$ & The channel from first transmit antenna to receive antenna \\
\hline$h_{2}$ & The channel from second transmit antenna to receive antenna \\
\hline$x_{1} \quad x_{2}$ & Transmitted symbols \\
\hline$n_{t} \quad n_{t+1}$ & Noise on first and second time slots \\
\hline
\end{tabular}

TABLE 2: Used Symbols Description

\section{BIT ERROR RATE}

In the proposed OFDM-SPM-BPSK-STBC system, two-bit sequences are transmitted. A bit can be received in error due to one of two possible reasons. First, a bit can be received with an error when detecting the power levels of the subcarriers is incorrect. This is because of the combined channel effects of both the noise and the multi-path fading. They can either amplify or attenuate the power of the subcarriers, leading to the detection of a high-power sub-carrier as a lowpower sub-carrier and vice versa. Second, an error can arise from the detection of the BPSK modulated symbols carried by the sub-carriers. Fig. 3 shows the higher power and lower power modulation bits constellation in the subcarriers. Since BPSK has only two levels, therefore, with sub-carrier power modulation, only one bit at a time can be transmitted.

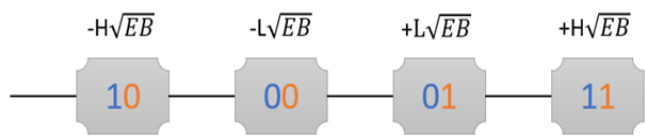

FIGURE 3: Constellation points of OFDM-SPM with BPSK

The BER equation for BPSK modulated symbols in the AWGN channel is given by

$$
B E R_{A W G N}=\frac{1}{2} \operatorname{erfc}\left(\sqrt{\frac{E_{b}}{N_{0}}}\right),
$$

here, $E_{b}=$ Energy per bit and $N_{0}=$ noise power spectral density. The probability that lower-power symbol detected as higher power symbol is given by [2]:

$$
\begin{aligned}
P_{L H}=\frac{1}{2} \operatorname{erfc}( & \left.\left(\frac{H-L}{2}\right) \sqrt{\frac{E_{b}}{N_{0}}}\right)+ \\
& \frac{1}{2} \operatorname{erfc}\left(\left(\frac{H+3 L}{2}\right) \sqrt{\frac{E_{b}}{N_{0}}}\right),
\end{aligned}
$$

similarly, the probability of occurrence of a high-power symbol being detected as a lower power symbol can be represented as:

$$
\begin{aligned}
P_{H L}=\frac{1}{2} \operatorname{erfc}( & \left.\left(\frac{H-L}{2}\right) \sqrt{\frac{E_{b}}{N_{0}}}\right)- \\
& \frac{1}{2} \operatorname{erfc}\left(\left(\frac{H+3 L}{2}\right) \sqrt{\frac{E_{b}}{N_{0}}}\right) .
\end{aligned}
$$

Hence, the actual BER for sub-carrier power bits can be given by:

$$
B E R_{P O W E R}=\frac{1}{2}\left(P_{L H}+P_{H L}\right) .
$$

The error probability for BPSK modulation bits is represented by:

$B E R_{B P S K}=\frac{1}{2}\left(\frac{1}{2}\left(1-\sqrt{\frac{L^{2}\left(\frac{E_{b}}{E_{0}}\right)}{1+L^{2}\left(\frac{E_{b}}{E_{0}}\right)}}+\left(\frac{1}{2}\left(1-\sqrt{\frac{H^{2}\left(\frac{E_{b}}{E_{0}}\right)}{1+H^{2}\left(\frac{E_{b}}{E_{0}}\right)}}\right)\right)\right.\right.$. 
The BER over multi path Rayleigh fading channel can be found by taking the PDF of Rayleigh fading and integrating it as follows.

$$
B E R_{\text {Ray-BPSK }}=\int_{0}^{\infty} B E R_{B P S K}(\gamma) f(\gamma) d \gamma .
$$

Here, $\gamma=\frac{E b}{N_{n}}$ and $f(\gamma)$ is the PDF of Rayleigh fading channel and is defined as $f(\gamma)=\frac{1}{\bar{\gamma}} e^{-\frac{\gamma}{\gamma}}$, where $\bar{\gamma}$ is the mean of $\gamma$.

The bit error rate for BPSK modulation in Rayleigh channel with Alamouti 2 transmit antenna, 1 receive antenna STBC case [22],

$$
p_{S T B C}=\frac{1}{2}-\frac{1}{2}\left(1+\frac{2}{\frac{E_{b}}{N_{0}}}\right)^{-\frac{1}{2}},
$$

and bit error rate (BER) is:

$$
P_{e, S T B C}=P_{S T B C}^{2}\left[1+2\left(1-p_{S T B C}\right)\right] .
$$

Since this design uses OFDM-SPM with BPSK as the modulation format, the overall equation for the bit error rate of this design is found by accounting for the Euclidean distances change caused by the high and low power levels of OFDMSPM-BPSK. As such, the overall bit error rate expression can be written, by manipulating (27), as follows:

$$
p_{S T B C-S P M}=\frac{1}{2}-\frac{1}{2}\left(1+\frac{2}{\left(\frac{H+L}{2}\right)^{2} \frac{E_{b}}{N_{0}}}\right)^{-\frac{1}{2}},
$$

where,

$$
\begin{gathered}
B E R_{S P M-S T B C, O V E R A L L}= \\
P_{S T B C-S P M}^{2}\left[1+2\left(1-p_{S P M-S T B C}\right)\right] .
\end{gathered}
$$

The results of the simulation are discussed next.

\section{RESULTS AND DISCUSSIONS}

In this section, simulation results of OFDM-SPM-STBC are discussed. The performance of the proposed design is quantified through the bit error rate and spectral efficiency metrics. All the simulations were conducted in the MATLAB simulation environment. The set of parameters involved in the simulation are shown in Table 3

The simulations have been done in different power policies, including the power saving policy (PSP) and the power

\begin{tabular}{|c|c|}
\hline Modulation Type & $B P S K(M=2)$ \\
\hline IFFT/FFT size & 64 \\
\hline Number of Active Subcarriers & 52 \\
\hline Cyclic prefix & 16 \\
\hline No of taps in the multi-path channel & 5 \\
\hline $\begin{array}{l}\text { Number of inactive sub-carriers for out of } \\
\text { band emission }\end{array}$ & 12 \\
\hline Number of frames & $2 \times 10^{4}$ \\
\hline Multi path channel delay samples location & {$\left[\begin{array}{lllll}0 & 3 & 5 & 6 & 8\end{array}\right]$} \\
\hline Multi path channel tap power profile $(\mathrm{dBm})$ & {$\left[\begin{array}{lllll}0 & -8 & -17 & -21 & -25\end{array}\right]$} \\
\hline Signal to Noise ratio (SNR in $\mathrm{dB}$ ) & 0 to $35 \mathrm{~dB}$ \\
\hline OFDM Bandwidth & $20 \mathrm{MHz}$ \\
\hline OFDM symbol duration & $4 \mu \mathrm{sec}$ \\
\hline Guard Interval & $0.8 \mu \mathrm{sec}$ \\
\hline
\end{tabular}
reassignment policy (PRP). Moreover, the modulation order is changed to an upper order to investigate the performance of the proposed design when higher modulation orders are used.
TABLE 3: Simulation Parameters.

\section{A. POWER SAVING POLICY}

OFDM-SPM aims to transmit more data bits per subcarrier by manipulating the power of the subcarriers in an OFDM block in addition to those bits that are usually transmitted by conventional modulation schemes. Due to this 2-D aspect of the OFDM-SPM modulation, transmitting the same number of bits OFDM-SPM requires only half the number of subcarriers needed by conventional OFDM. As such, different power policies were developed for OFDM-SPM. In the power saving policy (PSP), OFDM-SPM saves half the transmit power than conventional OFDM. The power of sub-carriers is saved to match the requirements of low power applications (e.g., IoT).

Simulation results of the bit error rate of OFDM-SPM-STBC in the power saving mode are shown in Fig 4

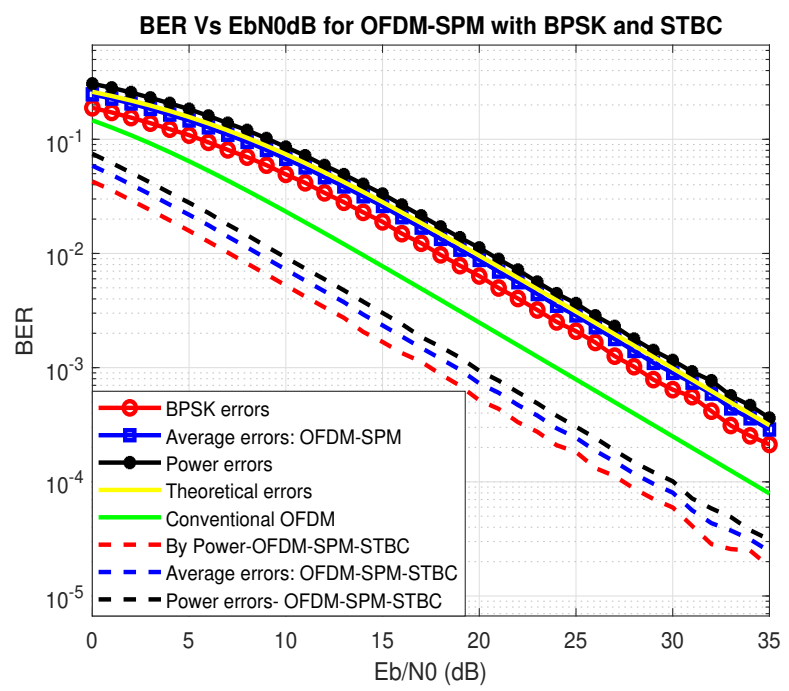

FIGURE 4: BER of OFDM-SPM-BPSK-STBC with power-saving policy.

We observe from Fig. 4 that the proposed system (OFDMSPM-BPSK-STBC) exhibits an advantage in the BER for the same SNR when compared with the conventional OFDMBPSK or OFDM-SPM-BPSK. For instance, at $20 \mathrm{~dB}$ SNR, 
the conventional OFDM-BPSK has a BER of around 0.0055, whereas the BER of OFDM-SPM-BPSK-STBC is approximately 0.001 and the BER for OFDM-SPM-BPSK without STBC is about 0.01 .

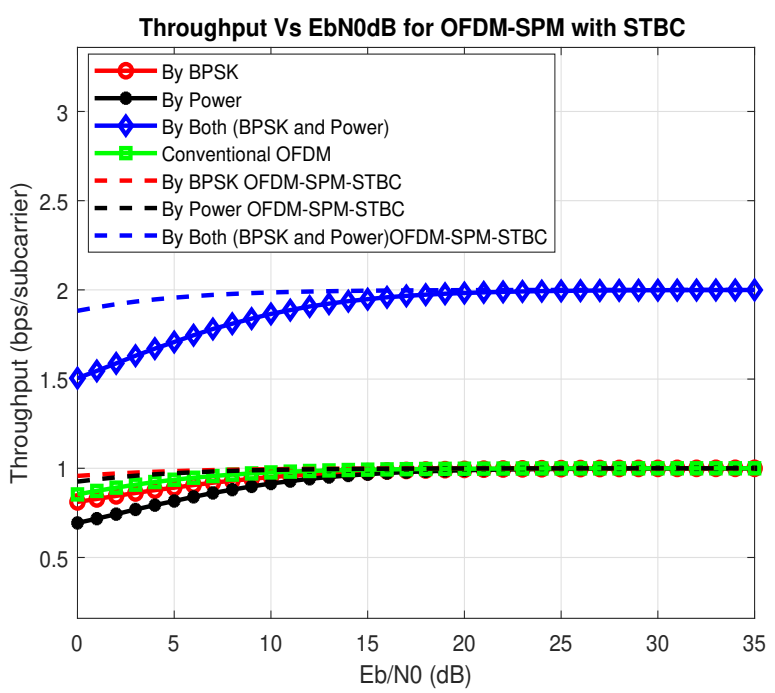

FIGURE 5: Throughput comparison for the proposed OFDMSPM-BPSK-STBC.

The throughput simulation results for this case are displayed in Fig. 5 where it is shown that the throughput is also better in the case of the proposed OFDM-SPM-BPSK-STBC at low SNR values. However, at higher SNR values, the signal power increases, and the noise level becomes low, and thus, STBC gain almost vanishes.

Table 4 shows the comparison between BER values for the power bits, BPSK bits, and average (average of the power and the BPSK bits) at different SNR points.

TABLE 4: BER comparison between OFDM-SPM and OFDMSPM-STBC at different SNR values for Power-saving policy.

\begin{tabular}{|c|c|c|c|}
\hline \multirow{2}{*}{ SNR Value } & \multicolumn{3}{|c|}{ OFDM-SPM } \\
\cline { 2 - 4 } & By BPSK & By Power & By Average \\
\hline \hline $10(\mathrm{~dB})$ & 0.04984 & 0.0861 & 0.06797 \\
\hline $15(\mathrm{~dB})$ & 0.01836 & 0.0326 & 0.02548 \\
\hline $20(\mathrm{~dB})$ & 0.006446 & 0.01137 & 0.008908 \\
\hline & \multicolumn{3}{|c|}{ OFDM-SPM-STBC } \\
\cline { 2 - 4 } SNR Value & By BPSK & By Power & By Average \\
\hline \hline $10(\mathrm{~dB})$ & 0.005322 & 0.009231 & 0.007276 \\
\hline $15(\mathrm{~dB})$ & 0.001707 & 0.003019 & 0.002363 \\
\hline $20(\mathrm{~dB})$ & 0.0005465 & 0.0009454 & 0.000746 \\
\hline
\end{tabular}

\section{B. POWER RE-ASSIGNMENT POLICY (PRP)}

In the reassignment power policy, the saved (i.e., unused) power is re-assigned to the transmit subcarriers. Two different cases of power reassignment policy for OFDM-SPMBPSK-STBC were simulated as discussed in the following sub-sections.

\section{1) Non-Optimized Power Re-assignment}

In the non-optimized power reassignment, the power saved is reassigned to the high-power subcarrier while the lower power subcarriers are set to unity.

The BER and throughput results for this case are plotted in Fig. 6 and Fig. 7, respectively.

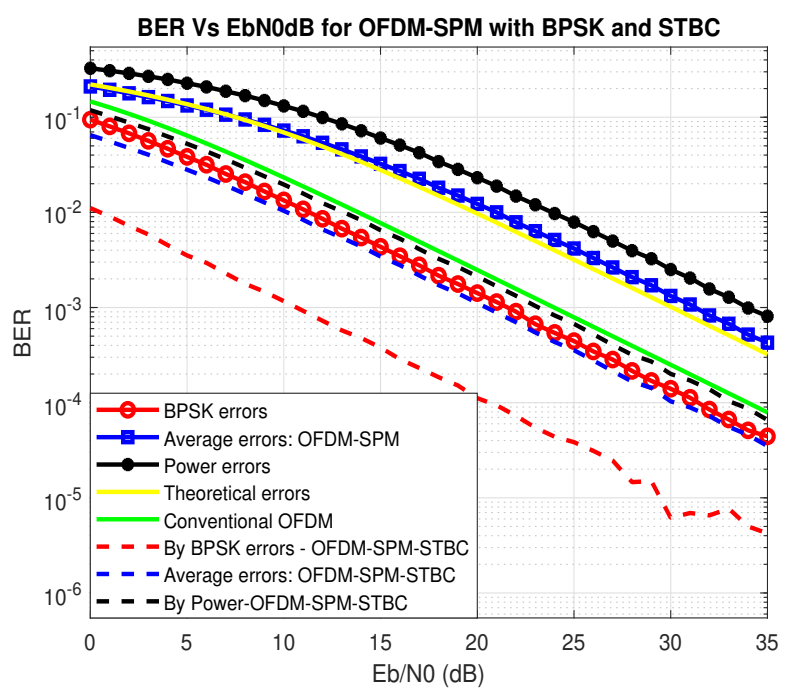

FIGURE 6: BER of OFDM-SPM-BPSK-STBC with nonoptimized power reallocation policy.

As can be observed, the BER for the proposed system (OFDM-SPM-BPSK-STBC) with power-reassignment nonoptimized policy has decreased for detecting the BPSK bits. In contrast, the BER for the power bits achieves the same performance as conventional OFDM without STBC. This case is concerned with improving the individual BPSK stream; however, the average curve adds slightly more performance gain in the BER than the power saving mode. Here, the diagram below shows the fact that applying the space-time block coding (STBC) technique in the case of non-optimized power policy makes good improvement in the BER. For example for a BER value of $10^{-3}$, we need an SNR value $\frac{E_{b}}{N_{0}}=10 \mathrm{~dB}$, While at the conventional model (OFDMSPM) we need $\frac{E_{b}}{N_{0}}=25 d B$ to achieve the same BER $10^{-3}$.

In a nut-shell, we analyze that this power-reassignment policy does not make much difference in terms of average BER improvement and throughout. Hence, it is better to use the power-saving policy than to use a non-optimized power assignment policy. The throughout in Fig.7 7 is almost similar as in the previous case of a power-saving policy. 


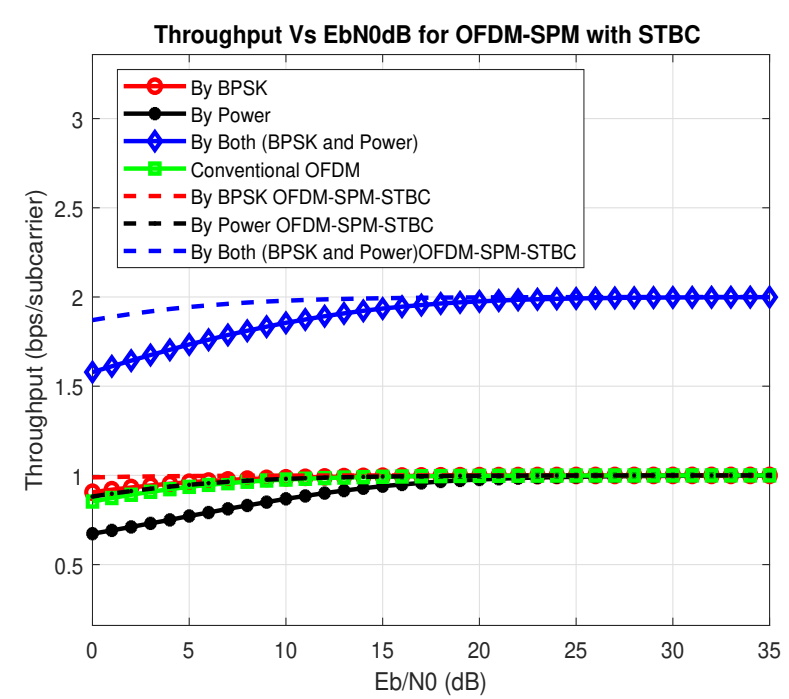

FIGURE 7: Throughput of OFDM-SPM-BPSK-STBC with nonoptimized power reallocation policy.

Table 5 shows the comparison between BER for power bits, BPSK bits, and average at different SNR points in the case of a non-optimized power-reassignment policy.

TABLE 5: BER comparison for OFDM-SPM and OFDMSPM-STBC at different value of SNR for non-optimized powerreassignment policy.

\begin{tabular}{|c|c|c|c|}
\hline \multirow{2}{*}{ SNR Value } & \multicolumn{3}{|c|}{ OFDM-SPM } \\
\cline { 2 - 4 } & By BPSK & By Power & By Average \\
\hline \hline $10(\mathrm{~dB})$ & 0.01337 & 0.1314 & 0.07225 \\
\hline $15(\mathrm{~dB})$ & 0.004345 & 0.06039 & 0.03237 \\
\hline $20(\mathrm{~dB})$ & 0.001418 & 0.02314 & 0.01228 \\
\hline \multirow{2}{*}{ SNR Value } & \multicolumn{3}{|c|}{ OFDM-SPM-STBC } \\
\cline { 2 - 4 } & By BPSK & By Power & By Average \\
\hline \hline $10(\mathrm{~dB})$ & 0.001159 & 0.01027 & 0.01938 \\
\hline $15(\mathrm{~dB})$ & 0.0003788 & 0.003435 & 0.006491 \\
\hline $20(\mathrm{~dB})$ & 0.00011115 & 0.001116 & 0.002121 \\
\hline
\end{tabular}

\section{2) Optimized Power Re-assignment}

In this case, the saved power is reassigned to the subcarriers so that the optimal high and low power levels are obtained for the average overall bit error rate. This optimization is done under a constraint that ensures that the average energy of an OFDM subcarrier in OFDM-SPM cannot exceed that of a subcarrier in conventional OFDM using BPSK symbol modulation as expressed by Eq.(2).

As can be observed from Fig. 8, the proposed model (OFDM-SPM-BPSK-STBC) exhibits excellent improvement in the BER when we implement an optimized power reassignment policy to the subcarriers. There is indeed an improvement in BPSK bits detection and power-bits detection, resulting in modifying the overall average bits detection. Compared to the conventional model OFDM-SPM, getting a $10^{-3}$ average BER we need $\frac{E_{b}}{N_{0}}=27.5 \mathrm{~dB}$, but only
$\frac{E_{b}}{N_{0}}=16 d B$ is needed using the proposed (OFDM-SPMSTBC) technique.

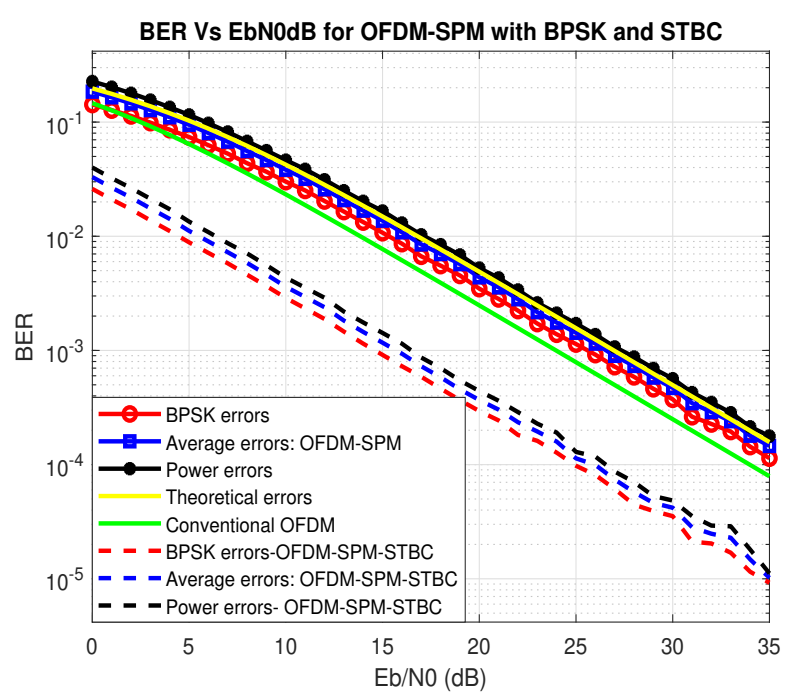

FIGURE 8: BER of OFDM-SPM-BPSK-STBC with optimized power reallocation policy.

The throughput of this case is plotted in Fig. 9 Table 6 shows a comparison between the proposed OFDM-SPMSTBC and the original scheme OFDM-SPM in the optimized power reassignment mode.

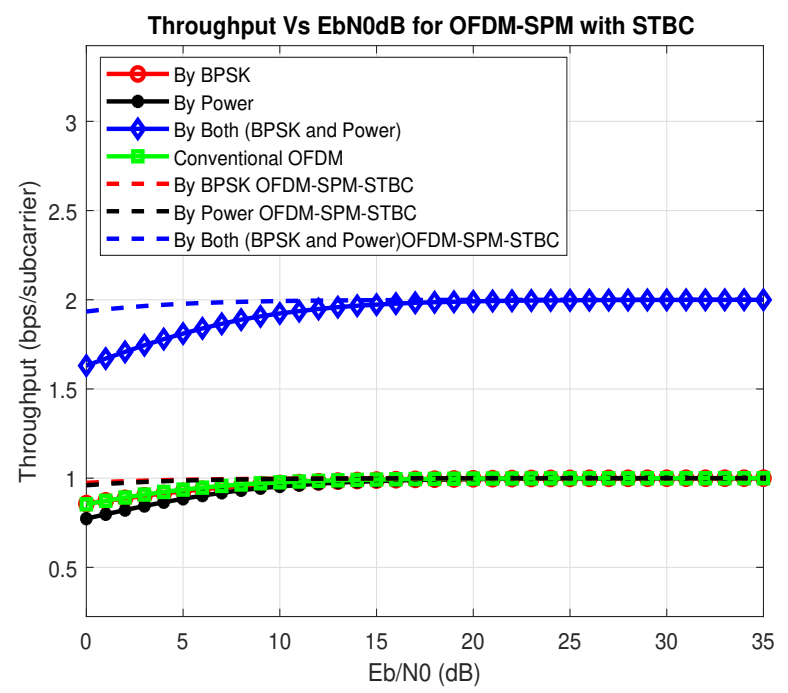

FIGURE 9: Throughput of OFDM-SPM-BPSK-STBC with optimized power reallocation policy.

The throughput plotted in Fig 9, is almost the same with no effect. The comparison of SNR is also drawn in Table 6 for the proposed OFDM-SPM-BPSK-STBC and non-STBC power bit and average bits detection. It can be seen that the 
average BER has improved in this case when compared to the power-saving and non-optimized case.

TABLE 6: BER comparison of OFDM-SPM-BPSK-STBC and OFDM-SPM-BPSK at different value of SNR for optimized power policy

\begin{tabular}{|c|c|c|c|}
\hline \multirow{2}{*}{ SNR Value } & \multicolumn{3}{|c|}{ OFDM-SPM } \\
\cline { 2 - 4 } & By BPSK & By Power & By Average \\
\hline \hline $10(\mathrm{~dB})$ & 0.03051 & 0.04687 & 0.03869 \\
\hline $15(\mathrm{~dB})$ & 0.01043 & 0.01614 & 0.01329 \\
\hline $20(\mathrm{~dB})$ & 0.003422 & 0.005264 & 0.004343 \\
\hline \multirow{3}{*}{ SNR Value } & By BPSK & By Power & By Average \\
\cline { 2 - 4 } & BFD-SPM-STBC \\
\hline \hline $10(\mathrm{~dB})$ & 0.002834 & 0.004507 & 0.003671 \\
\hline $15(\mathrm{~dB})$ & 0.0008946 & 0.001427 & 0.001161 \\
\hline $20(\mathrm{~dB})$ & 0.0002877 & 0.0007396 & 0.0003637 \\
\hline
\end{tabular}

\section{CONCLUSION AND FUTURE SCOPE}

In this paper, OFDM with Subcarrier Power Modulation (OFDM-SPM) was studied with Alamouti space-time block coding (STBC) in Rayleigh fading channels. The proposed technique was simulated under different power scenarios, including the power saving mode and the power reassignment mode. It was found that OFDM-SPM-STBC enhances the reliability performance of the original OFDM-SPM. We implement the proposed scheme with power-saving and powerreassignment policies of SPM. We show that the powerreassignment with an optimized power allocation scheme has the most significant improvement in the BER.

In contrast, the power-saving SPM scheme has the intermediate BER improvement, and non-optimized power reassignment has the least. As a future research direction, we can implement this proposed OFDM-SPM-STBC scheme with higher constellation modulation schemes and also analyze the complexity and sensitivity of the equalizer and receiver. The sensitivity and complexity analysis would be of considerable interest because with higher constellation modulation, a more significant number of sub-carrier power levels would be required. The detection of those sub-carrier power levels may need more sensitive receivers to add complexity to the system. We can also formulate the multi-objective optimization problem for the trade-off between the sensitivity and complexity of the OFDM-SPM-STBC receiver.

\section{REFERENCES}

[1] C. Chung, "Spectral preceding for constant-envelope OFDM", IEEE Transaction on Communications, Vol. 58, No. 6, pp. 555 - 567, 2010.

[2] J. M. Hamamreh, and A. Hajar, "The Generalization of Orthogonal Frequency Division Multiplexing with Subcarrier Power Modulation to Quadrature Signal Constellations", RS Open Journal on Innovative Communication Technologies, 1(1), 2020, https://doi.org/10.21428/03d8ffbd.4948e89e.

[3] S. M. Alamouti, "A simple transmit diversity technique for wireless communications," in IEEE Journal on Selected Areas in Communications, vol. 16, no. 8, pp. 1451-1458, Oct. 1998, doi: 10.1109/49.730453.

[4] J. M. Hamamreh, Abdulwahab Hajar, and Mohamedou Abewa, "Orthogonal Frequency Division Multiplexing With Subcarrier Power Modulation for Doubling the Spectral Efficiency of 6G and Beyond Networks." in Transactions on Emerging Telecommunications Technologies, 2020.
[5] Abewa, M., Hamamreh, J. M., Non-coherent OFDM-Subcarrier Power Modulation for Low Complexity and High Throughput IoT Applications. RS Open Journal on Innovative Communication Technologies, 1(1), 2020 https://doi.org/10.46470/03d8ffbd.2a45a9a1

[6] Youcef Belallou, Jehad M. Hamamreh, and Abdulwahab Hajar, "OFDMSubcarrier Power Modulation With Two Dimensional Signal Constellation," 2019 IEEE Conference on Innovations in Intelligent Systems and Applications (ASYU 2019), Izmir, 2019, pp. 1-6.

[7] Abdulwahab Hajar, Jehad M. Hamamreh, Mohamedou Abewa, and Youcef Belallou, "A Spectrally Efficient OFDM-Based Modulation Scheme for Future Wireless Systems," 2019 IEEE Electric Electronics, Computer Science, Biomedical Engineerings' Meeting (EBBT), Istanbul, 2019, pp. $1-4$.

[8] A. Kamil and M A Hadi, "STBC-OFDM System under Rayleigh Fading Channel with Known and Unknown CSI at the Transmitter", International Journal of Innovative Research in Science, Engineering and Technology, Vol. 7, No. 5, pp. 5377-5384, 2018, doi:10.15680/IJIRSET.2018.0705113.

[9] J. Yang and K. Cheun, "Low complexity implementation of Alamouti space-time coded OFDM transmitters," in IEEE Communications Letters, vol. 8, no. 4, pp. 229-231, April 2004, doi: 10.1109/LCOMM.2004.827443.

[10] F. A. P. de Figueiredo, N. F. T. Aniceto, J. Seki, I. Moerman and G. Fraidenraich, "Comparing f-OFDM and OFDM Performance for MIMO Systems Considering a 5G Scenario," 2019 IEEE 2nd 5G World Forum (5GWF), Dresden, Germany, 2019, pp. 532-535, doi 10.1109/5GWF.2019.8911702.

[11] Y. J. Moon et al., "OFDM-based 25Gbps Wireless Backhaul System for 5G Convergence Service," 2020 International Conference on Information Networking (ICOIN), Barcelona, Spain, 2020, pp. 814-817, doi: 10.1109/ICOIN48656.2020.9016621.

[12] A. Molisch, Wireless Communications. Wiley-IEEE Press, 2005.

[13] J. Winters, "On the capacity of radio communication systems with diversity in a Rayleigh fading environment," IEEE Journal on Selected Areas in Communications, vol. 5, no. 5, pp. 871-878, 1987.

[14] P. Pathak, R. Pandey, "A Novel Alamouti STBC Technique for MIMO System Using 16-QAM Modulation and Moving Average Filter", Int. Journal of Engineering Research and Applications, Vol. 4, No. 8, August 2014, pp.49-55.

[15] P. Zetterberg, M. L. Nordenvaad and B. Nilsson, "Synchronization of OFDM with null subcarriers in channels with significant ICI," OCEANS 2017 - Aberdeen, Aberdeen, 2017, pp. 1-5, doi 10.1109/OCEANSE.2017.8084727.

[16] K.V.N. Kavitha, S. Ghosh, A. Keeley, S. Khara, "Error Rate Analysis of STBC-OFDM System with Efficient Channel Coding Technique at low SNR," International Journal of Applied Engineering Research, Vol 9, No. 16, 2014, pp. 3481-3494.

[17] J. P. Singh and S. Singh,quot;Implementation of OFDM and other multicarrier modulations on SDR,quot; 2016 IEEE International Conference on Signal Processing, Communication, Power and Embedded System (SCOPES), Paralakhemundi, 2016, pp. 1937-1940.

[18] J. M. Hamamreh, E. Basar, and H. Arslan, "OFDM-subcarrier index selection for enhancing security and reliability of 5G URLLC services," IEEE Access, vol. 5, pp. 25 863-25 875, 2017.

[19] Fiorina, Jocelyn. (2012). A New Family of Low-Complexity Decodable STBCs for 4 Transmit Antennas. IEEE Transactions on Wireless Communications. 12. 10.1109/TWC.2013.011513.120517.

[20] Y. Belallou, J. M. Hamamreh and A. Hajar, "OFDM-Subcarrier Power Modulation with two-dimensional signal constellations," 2019 Innovations in Intelligent Systems and Applications Conference (ASYU), Izmir, Turkey, 2019, pp. 1-6, doi: 10.1109/ASYU48272.2019.8946346.

[21] R. Abu-alhiga and H. Haas, "Subcarrier-index modulation OFDM," in 2009 IEEE 20th International Symposium onPersonal, Indoor and Mobile Radio Communications, Sep. 2009, pp. 177-181.

[22] Maximal Ratio Combining: http://www.dsplog.com/2008/09/28/maximalratio-combining.

[23] Abewa, M., Hamamreh, J. M. (2021). NC-OFDM-SPM: A TwoDimensional Non-Coherent Modulation Scheme for Achieving the Coherent Performance of OFDM along with Sending an Additional Datastream. RS Open Journal on Innovative Communication Technologies, 2(3). https://doi.org/10.46470/03d8ffbd.a97a5236.

[24] Hajar, A., Hamamreh, J. M. (2020). The Generalization of Orthogonal Frequency Division Multiplexing With Subcarrier Power Modulation to Quadrature Signal Constellations. RS 
Open Journal on Innovative Communication Technologies, 1(1). https://doi.org/10.21428/03d8ffbd.4948e89e.

[25] M. F. Zia and J. M. Hamamreh, "An Advanced NOMA Security Technique for Future Wireless Communication," Workshop on Information and Communications Technologies, International Conference on Software, Telecommunications and Computer Networks (SoftCOM), Sep. (2020), pp. 38-43.

[26] Lemayian, J. P., Hamamreh, J. M. (2020). A Novel Small-Scale Nonorthogonal Communication Technique Using Auxiliary Signal Superposition with Enhanced Security for Future Wireless Networks. RS Open Journal on Innovative Communication Technologies, 1(2). https://doi.org/10.46470/03d8ffbd.86b0d106.

[27] Hamamreh, J. M., Abewa, M., Lemayian, J. P. (2020). New Non-Orthogonal Transmission Schemes for Achieving Highly Efficient, Reliable, and Secure Multi-User Communications. RS Open Journal on Innovative Communication Technologies, 1(2). https://doi.org/10.46470/03d8ffbd.324cc0fb

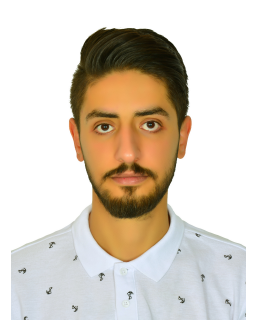

ABDELRAHMAN M.A. ABUQAMAR received the B.Sc. degree in mechatronics engineering from Philadelphia University, Jordan, in 2018.

$\mathrm{He}$ is the co-author of First publications and has worked with professor Jehad M. Hamamreh on the topic of OFDM-SPM-STBC, also he is studying for a masters degree In the department of electrical and computer engineering, Antalya Bilim University in Antalya, Turkey

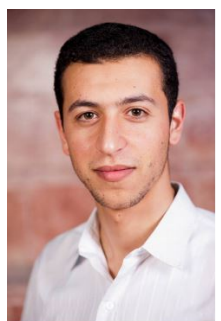

JEHAD M. HAMAMREH received the B.Sc. degree in electrical and telecommunication engineering from An-Najah University, Nablus, in 2013, and the Ph.D. degree in electrical-electronics engineering and cyber systems from Istanbul Medipol University, Turkey, in 2018. He was a Researcher with the Department of Electrical and Computer Engineering, Texas A and M University. He is currently an Assistant Professor with the Electrical and Electronics Engineering Department, Antalya International (Bilim) University, Turkey. His current research interests include wireless physical and MAC layers security, orthogonal frequency-division multiplexing multiple-input multiple-output systems, advanced waveforms design, multi-dimensional modulation techniques, and orthogonal/non-orthogonal multiple access schemes for future wireless systems. He is a regular investigator and referee for various scientific journals as well as a TPC Member for several international conferences. He can be reached via e-mail: jehad.hamamreh@gmail.com // web: https://sites.google.com/view/wislab.

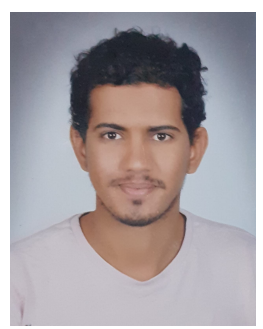

MOHAMEDOU ABEWA received his B.Sc. degree in Electrical and Electronics Engineering from Antalya Bilim University, Turkey in 2019. Currently, he is pursuing a master's degree in Electrical and Computer Engineering at the same university. He is pursuing his research/thesis work at the Wireless Intelligent Systems Laboratory (WISLAB) at Antalya Bilim University. His research interests include Orthogonal Frequency Division Multiplexing (OFDM) related waveform designs, physical layer security algorithms and Non-Orthogonal Multiple Access Techniques (NOMA) for future wireless systems. 